\title{
Restricciones institucionales que dificultan la modelización espacio-geométrica en la enseñanza secundaria
}

\author{
Carlos Rojas, Universidad de Antioquia (Colombia) \\ Tomás Ángel Sierra, Universidad Complutense de Madrid (España)
}

\begin{abstract}
Restricciones institucionales que dificultan la modelización espacio-geométrica en la enseñanza secundaria

\section{Resumen}

Presentamos restricciones institucionales que dificultan la modelización espacio-geométrica en la enseñanza secundaria. Con el objetivo de explicarlas, describimos y analizamos tanto la actividad matemática propuesta sobre construcción de cuerpos geométricos en el manual utilizado por alumnos de $3 .^{\circ}$ de Educación Secundaria Obligatoria como la experimentada en un recorrido de estudio e investigación sobre un problema espacial acerca del diseño y construcción de envases. Utilizamos elementos basados en los indicadores del grado de completitud de una organización matemática local que caracterizan los tipos de tareas de modelización matemática. Los resultados indican, principalmente, que, aunque el tipo de problema propuesto posee potencial para provocar la necesidad de utilizar procesos de modelización y para dar sentido a saberes curriculares sobre la determinación y construcción de cuerpos geométricos, el impacto del currículo dominante al que se enfrentan diariamente los estudiantes dificulta dichos procesos.
\end{abstract}

Palabras clave. Modelización espacio-geométrica; construcción de sólidos; restricciones institucionales; educación secundaria; recorrido de estudio e investigación.

Institutional constraints that hinder spatial-geometric modelling in secondary school teaching

\section{Abstract}

We present institutional restrictions that hinder spatial-geometric modelling in secondary school teaching. With the aim to explain them, we describe and analyse the mathematical activity about the construction of geometric bodies in the textbook used by students in the 3rd year of Compulsory Secondary Education, and the activity experienced in a study and research path on a spatial problem about the design and construction of containers. We use elements based on the indicators of the degree of completeness of a local mathematical organisation that characterise the types of mathematical modelling tasks. The results mainly show that, although the type of problem proposed has the potential to provoke the need to use modelling processes and to make sense of curricular knowledge on the determination and construction of geometric bodies, the impact of the dominant curriculum that students daily face hinders such processes.

Keywords. Spatial-geometric modelling; construction of solids; institutional constraints; secondary education; study and research path.

\section{Introducción y presentación de la problemática}

El desarrollo y análisis de procesos de enseñanza y aprendizaje, donde se trata de implementar técnicas de modelización matemática, es un dominio de investigación de gran interés en didáctica de las matemáticas (Barquero, 2020; Schukajlow et al., 2018). En línea con dicho dominio, nuestro trabajo pretende mostrar, analizar y explicar las restricciones y limitaciones que se presentan en la Educación Secundaria Obligatoria (ESO), para que los alumnos utilicen técnicas de modelización matemática cuando se les proponen problemas en torno a la determinación y construcción de sólidos.

Postulamos que dichas restricciones configuran un fenómeno didáctico de origen institucional y que, en rigor, no deberían considerarse dificultades de los alumnos para utilizar adecuadamente dichas técnicas. Se trata de dificultades del propio sistema educativo 
para poner a disposición de los alumnos dichas técnicas. En definitiva, pretendemos abordar la dimensión ecológica del problema de investigación (Barquero et al., 2013) que surge a partir del problema docente siguiente: ¿Cómo enseñar geometría 3D utilizando para ello técnicas de modelización?

Según Perrin-Glorian et al. (2013), una manera de conseguir una enseñanza coherente y funcional de la geometría 3D es mediante lo que Berthelot y Salin (2005) llaman problemática de modelización del espacio o problemática espacio-geométrica. Se parte de un problema espacial o del espacio sensible que se pasa a representar mediante un modelo geométrico en el que, utilizando sus propiedades geométricas, se obtiene una solución, que termina validándose en el espacio sensible.

Además, la búsqueda de posibles respuestas al problema espacial puede enriquecerse utilizando tecnicas de modelización algebraico-funcionales mediante un programa de matemáticas dinámicas como Geogebra o de diseño de piezas en 3D como Tinkercad, lo que permitirá articular el estudio de la geometría 3D con el del álgebra y las funciones.

En el apartado 2, presentaremos, en primer lugar, en qué consiste la problemática de la modelización espacio-geométrica, como un tipo particular de modelización matemática (sección 2.1). En segundo lugar, abordaremos cómo es concebida la modelización matemática en la teoría antropológica de lo didáctico (TAD), enfoque didáctico que utilizaremos como modelo teórico, metodológico y analítico fundamental para nuestro estudio (sección 2.2). Luego, mostraremos las características del dispositivo didáctico que propone la TAD para hacer posible el desarrollo de técnicas de modelización (sección 2.3). En el apartado 3, presentaremos el problema de investigación y la metodología seguida. Posteriormente, describiremos y analizaremos los tipos de problemas y técnicas que aparecen en el libro de texto utilizado por los alumnos (apartado 4). Ello nos permitirá identificar y explicar restricciones institucionales que afectan a la actividad de modelización (apartado 5). En el apartado 6, explicaremos y analizaremos el desarrollo de un recorrido de estudio e investigación (REI) propuesto a los estudiantes, centrado en la determinación y construcción de objetos y diseñado como una propuesta alternativa para hacer vivir un verdadero proceso de modelización espacio-geométrica en la educación secundaria. Terminaremos analizando hasta qué punto persisten algunas de las restricciones y limitaciones detectadas para abordar técnicas de modelización matemática, a pesar de haber implementado un proceso de estudio que favorece su desarrollo (apartado 7), y exponiendo algunas conclusiones (apartado 8).

\section{Marco teórico y antecedentes}

\subsection{La modelización espacio-geométrica}

La noción de modelización espacio-geométrica (Berthelot y Salin, 2005) describe una relación entre el espacio sensible y los saberes geométricos donde, al resolver un tipo de problemas, los problemas espaciales, dichos saberes aparecen como necesarios y se convierten en herramientas útiles para su resolución. Utilizamos la definición de problemas espaciales que propone Salin (2004), donde los caracteriza porque:

Su finalidad concierne al espacio sensible [y] pueden tratar sobre la realización de: acciones

[como] fabricar, desplazarse, desplazar, dibujar, etc., [y sobre] comunicaciones a propósito de acciones o de constataciones [...] [Además, porque] el éxito o el fracaso viene determinado para el individuo por la comparación entre el resultado esperado y el resultado obtenido. (p. 39) 
La problemática espacio-geométrica que nosotros consideramos para el caso de la determinación y construcción de sólidos parte de un sistema donde se plantea un tipo de problemas espaciales. Para resolver dichos problemas se elabora un modelo matemático adecuado que representa dicho sistema, en el que se utilizan elementos matemáticos de todo tipo (geométricos, aritméticos, algebraicos, funcionales, etc.). Se trabaja y se construyen respuestas en dicho modelo a las cuestiones planteadas y se termina validando las respuestas obtenidas en el espacio sensible. Se trata de proponer el estudio de la geometría como modelo del espacio, tal como señala Brousseau (citado en Berthelot y Salin, 2001) cuando plantea una situación fundamental para la geometría elemental como modelo del espacio. Brousseau explica que, para generar situaciones a-didácticas cuyo objetivo sea la elaboración de conocimientos geométricos, se puede acudir a algunos ejemplos entre los que está la determinación y realización de sólidos.

En la problemática de la modelización espacio-geométrica se necesita que la búsqueda de posibles respuestas no se agote en las soluciones de un caso particular (Salin, 2004). Por ejemplo, si se quiere construir un cristal para una ventana con una forma particular de paralelogramo, ¿qué técnicas podrían utilizarse? Una técnica podría consistir en realizar una copia del cristal con un papel adecuado, utilizando conocimientos espaciales relativos a la orientación del papel y del cristal, a la manipulación de herramientas del cristalero para cortar el cristal, etc. Otra técnica más eficaz sería emplear las características que definen un paralelogramo como, por ejemplo, que sus diagonales se cortan en el punto medio de ambas. Esta última será la solución del problema por modelización, ya que se construye totalmente dentro del sistema simbólico del modelo.

\subsection{La modelización matemática en la teoría antropológica de lo didáctico}

La modelización espacio-geométrica se puede insertar en la problemática más amplia de los procesos de modelización y su relación con la enseñanza y el aprendizaje de las matemáticas. Esta problemática ha sido ampliamente abordada desde la TAD. En esta teoría, la modelización se puede describir en cuatro estadios: 1) se parte del planteamiento de una situación y problemática inicial, que bien puede ser extra-matemática o intra-matemática. Este estadio se caracteriza por la construcción del sistema a modelizar y por el planteamiento de cuestiones amplias que surgen ante la imposibilidad de darles una respuesta inmediata; 2) se elabora un modelo matemático del sistema construido y se definen y relacionan las variables pertinentes; 3 ) se manipula y refina el modelo elaborado, y se interpretan sus resultados a la luz del sistema modelizado; y 4) se formulan cuestiones nuevas que aportan al estudio del sistema o que conducen a nuevos procesos de modelización (Florensa et al., 2020; Gascón, 1994). Así, la forma en que la TAD considera la modelización matemática tiene tres características diferenciales: a) incorpora la modelización intra-matemática como un caso importante de modelización matemática; b) los modelos que se construyen tienen estructura praxeológica y se consideran "máquinas" o herramientas que permiten aportar conocimientos y plantear cuestiones sobre el sistema objeto de estudio; y c) dicho proceso consiste en la elaboración de praxeologías de complejidad creciente que permitirá articular diferentes dominios de la actividad matemática escolar (Barquero et al., 2013).

En trabajos anteriores, realizados en el marco de la TAD, se han estudiado diferentes aspectos del fenómeno didáctico que consiste en el debilitamiento de la actividad de modelización matemática, tal como se manifiesta en diferentes instituciones escolares. Por ejemplo, Fonseca (2004) estudió la rigidez, desarticulación e incompletitud de las organizaciones matemáticas escolares en el paso de secundaria a la universidad. Barquero (2009) analizó la desarticulación entre las matemáticas enseñadas y las ciencias 
experimentales en el primer curso universitario que degenera en un mero aplicacionismo, y Serrano (2013) llevó a cabo un estudio similar en el caso de las ciencias empresariales. Otros trabajos han estudiado la manifestación de dicho fenómeno disciplinar en diferentes dominios específicos de la matemática escolar. Por ejemplo, García (2005) estudió la desarticulación de la relación de proporcionalidad y el resto de relaciones funcionales que se estudian en secundaria. Ruiz-Munzón (2010) analizó las consecuencias de considerar el álgebra elemental en secundaria como una aritmética generalizada y elaboró, siguiendo los trabajos de Bolea (2002), un modelo epistemológico alternativo del álgebra elemental interpretándola como un instrumento de modelización y su prolongación hacia la modelización algebraico-funcional. Y Lucas (2015) estudió el fenómeno de la pérdida de las razones de ser del cálculo diferencial en el paso de la secundaria a la universidad que se presenta ligado a la ausencia de una problemática de modelización funcional.

En nuestra investigación, centrada en la geometría, tras revisar y analizar en el currículo de la ESO algunos libros de texto (Rojas y Sierra, 2017), encontramos rasgos del fenómeno didáctico general descrito anteriormente. En concreto, hemos observado la desarticulación existente entre la geometría 2D y 3D, así como la rigidez e incompletitud de las organizaciones geométricas escolares. En Rojas y Sierra (2021) hemos mostrado que, para resolver problemas geométricos, se presentan las diferentes técnicas de forma aislada y se hace un uso casi exclusivamente aritmético de las fórmulas del cálculo de áreas y volúmenes, tratándolas como meros algoritmos de cálculo, porque los tipos de tareas propuestos escolarmente así lo demandan. De hecho, sucede que los tipos de tareas geométricas que se proponen en los manuales escolares vienen justificados por las técnicas que se desea sean empleadas para su resolución, y viceversa. En resumen, se pone de manifiesto un «empobrecimiento» de las razones de ser de la enseñanza de la geometría en la educación secundaria si la comparamos con una posible razón de ser basada en la modelización espacio-geométrica.

En este trabajo, pretendemos seguir analizando el fenómeno didáctico del debilitamiento de la actividad de modelización en el ámbito de la geometría, sabiendo que no debe considerarse como un fenómeno aislado. Además, queremos avanzar en el estudio del origen de las dificultades de los alumnos de la ESO cuando se enfrentan a problemas en torno a la determinación y construcción de sólidos, que proponemos como una posible razón de ser de la geometría escolar.

\subsection{Los recorridos de estudio e investigación y la actividad de modelización}

Dentro de la TAD se han desarrollado los recorridos de estudio e investigación (REI), como dispositivos especialmente diseñados para hacer posible la vida escolar de las actividades de modelización matemática (Barquero, 2009; Florensa et al., 2020). En un REI se parte de una cuestión viva y fértil, de interés para la comunidad de estudio que la abordará y para la que no tiene una respuesta inmediata. Durante la búsqueda de una respuesta plausible a dicha cuestión, surgirán algunas respuestas parciales y otras cuestiones derivadas, cuyo estudio ayudará a que la comunidad de la clase adquiera un conocimiento funcional en torno a un ámbito determinado de las matemáticas que servirá para dar respuesta a las cuestiones planteadas.

En coherencia con el postulado de la TAD que identifica la actividad matemática con la actividad de modelización, hemos planteado un REI en torno a cuestiones sobre el diseño y construcción de un envase con una determinada capacidad con un objetivo doble: por un lado, analizar la habilidad de los alumnos para construir, partiendo de sus conocimientos escolares, técnicas de modelización espacio-geométrica útiles para resolver este tipo de problemas y, por otro, experimentar hasta qué punto la actividad 
matemática desarrollada en dicho proceso de estudio permite superar alguno de los efectos que producen sobre el aprendizaje de los alumnos las restricciones y limitaciones provocadas por el tipo de actividad matemática propuesta en el manual escolar.

\section{Problema de investigación y metodología}

La investigación que realizamos es de tipo cualitativo basada en el estudio de casos, donde se analiza, por un lado, el manual que utilizan habitualmente los estudiantes y, por otro, la actividad matemática que llevan a cabo dichos estudiantes en la experimentación de un REI. Nuestro problema de investigación puede desglosarse en tres aspectos:

a) Detectar y explicar las restricciones institucionales que dificultan y limitan la posibilidad de que los alumnos de educación secundaria puedan poner en práctica técnicas de modelización espacio-geométrica y algebraico-funcionales.

b) Diseñar un REI para indagar en qué medida su implementación favorece que los alumnos de secundaria construyan técnicas de modelización espacio-geométrica y algebraico-funcionales.

c) Valorar hasta qué punto el REI experimentado puede facilitar la superación de algunas de las restricciones institucionales presentadas en el análisis del manual escolar, y detectar aquellas que son persistentes y las nuevas que puedan aparecer.

Basaremos la explicación y análisis de las diferentes restricciones institucionales que dificultan e incluso impiden que los alumnos de la ESO utilicen técnicas de modelización para abordar la construcción y determinación de sólidos, en el contraste entre:

a) La descripción y análisis breve de la actividad matemática en torno a la construcción de cuerpos geométricos que se propone en el manual escolar, utilizado por los alumnos en sus clases ordinarias de matemáticas. En concreto, nos centraremos en la unidad 9 del libro de matemáticas orientadas a las enseñanzas académicas de $3 .^{\circ}$ de la ESO, de la editorial SM Savia, titulada Cuerpos geométricos.

b) La descripción, análisis y explicación del tipo de tareas y técnicas que utilizan los alumnos cuando se enfrentan a un problema espacial y, en particular, el análisis de las dificultades que encuentran para utilizar la modelización matemática cuando se enfrentan al problema de diseñar y construir envases.

\section{Descripción y análisis del proceso de estudio propuesto en el libro de texto}

\subsection{Descripción del proceso de estudio}

El texto escolar, utilizado por los alumnos, contiene 14 unidades y la novena es la titulada Cuerpos geométricos. Dicha unidad presenta 7 secciones; las que se refieren de modo más específico a la construcción de cuerpos geométricos son las siguientes:

- En la sección 2, se define y muestra qué es un poliedro y se presenta la fórmula de Euler como una igualdad que cumplen todos (sic) los poliedros. Después se muestra y define qué es un prisma y qué es una pirámide con sus respectivos elementos. Se termina definiendo qué es un poliedro regular y mostrando los poliedros regulares que existen.

- En la sección 3, se define qué es un cuerpo de revolución y se muestran mediante una representación plana el cilindro, el cono, el tronco de cono y la esfera, indicando cómo se obtienen. La sección finaliza mostrando los elementos y partes de una esfera. 
- En la sección 4, se definen área lateral, área total y volumen de un prisma, de una pirámide, de un cilindro, de un cono, de una esfera y de las partes de una esfera, mostrando una representación plana del sólido en cuestión y un desarrollo plano cuando es posible.

- En la sección 5, se muestra cómo calcular el volumen de otros cuerpos geométricos, formados por la unión de varios cuerpos simples (ver Tabla 1).

Tabla 1. Tipos de tareas presentes en la sección 5 (Alcaide et al., 2016, p. 200)

\begin{tabular}{ll}
\hline \multicolumn{1}{c}{ Tipo de tarea } & \multicolumn{1}{c}{ Técnica empleada } \\
\hline $\begin{array}{l}\text { Calcular área y volumen } \\
\text { de sólidos compuestos }\end{array}$ & $\begin{array}{l}\text { Dividir el cuerpo compuesto en varios simples y sustituir los valores de } \\
\text { cada cuerpo en sus fórmulas del área y del volumen y sumar los valores } \\
\text { por otros sólidos simples. }\end{array}$ \\
obtenidos, sin considerar las áreas de las caras de las uniones.
\end{tabular}

Ejemplo de actividad resuelta en p. 200

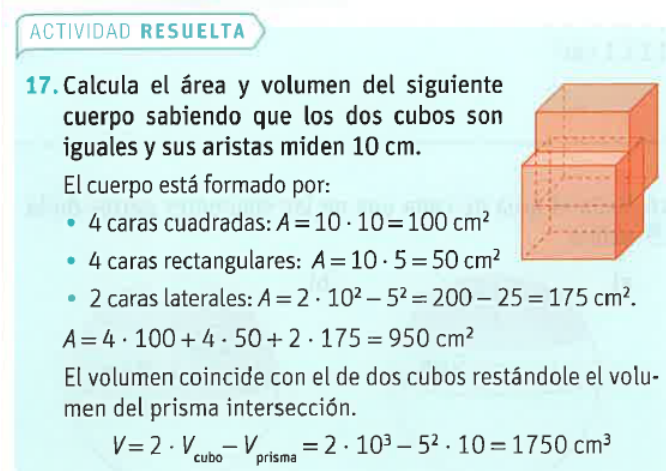

Para terminar la unidad se proponen 44 actividades para resolver, de las que 22 tratan sobre el cálculo de áreas y volúmenes de un cuerpo geométrico. En todos los casos se proporcionan los datos necesarios que, al sustituirlos en la fórmula correspondiente, permiten mediante un cálculo aritmético obtener el resultado pedido. Los datos en algunos casos se dan directamente y otras veces se indican en una representación plana de la figura. En algunas ocasiones se pide realizar un desarrollo plano del cuerpo geométrico correspondiente. Sólo hay tres ejemplos en los que se plantea, de forma un poco anecdótica, una tarea inversa. En estos, o se proporciona el valor del área total del cuerpo geométrico y se pide calcular el volumen, o se proporciona el volumen y se pide calcular el área total, aunque, como en el caso de la Figura 1, los tres tienen solución única:

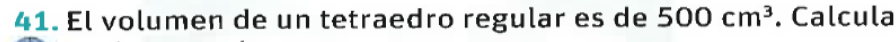

Figura 1. Tarea inversa propuesta en el libro de texto (Alcaide et al., 2016, p. 207)

En general, los autores, siempre que proponen una actividad con más dificultad, optan por resolverla y, de este modo, los alumnos pueden imitar el proceso seguido para resolver las siguientes. El capítulo termina con una autoevaluación con 9 actividades y en 4 de ellas se pide calcular el área, el volumen o el desarrollo plano de un cuerpo geométrico.

\subsection{Análisis del proceso de estudio propuesto en el texto escolar}

El tipo de tareas que aparecen en el libro de texto son, como las de las Figuras 2 y 3 , de naturaleza directa y de respuesta única. Para resolverlas basta sustituir las medidas de las longitudes proporcionadas en las fórmulas correspondientes, lo que conlleva un uso eminentemente aritmético y algorítmico de las fórmulas. 


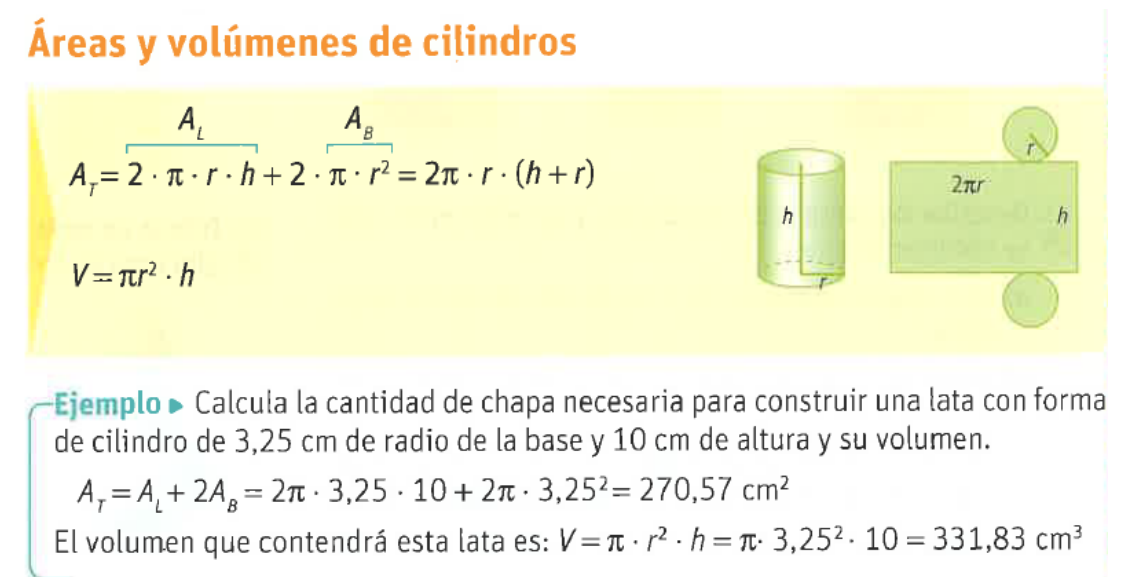

Figura 2. Ejemplo de cálculo de áreas y volúmenes de cilindros (Alcaide et al., 2016, p. 198)

$$
\begin{aligned}
& \text { ACTIVIDADES } \\
& \text { 15. Calcula el área total y el volumen de estos cuerpos. } \\
& \text { a) Un cubo de } 10 \mathrm{~cm} \text { de arista. } \\
& \text { b) Un prisma regular de } 10 \mathrm{~cm} \text { de altura, cuya base es un } \\
& \text { cuadrado de } 20 \mathrm{~cm} \text { de lado. } \\
& \text { c) Un cilindro de } 10 \mathrm{~cm} \text { de radio de la base y altura igual al } \\
& \text { diámetro de la base. } \\
& \text { d) Un cono de } 8 \mathrm{~cm} \text { de radio de la base y } 16 \mathrm{~cm} \text { de altura. } \\
& \text { e) Una esfera de } 10 \mathrm{~m} \text { de diámetro. }
\end{aligned}
$$

Figura 3. Actividades de cálculo de áreas y volúmenes (Alcaide et al., 2016, p. 199)

Una posible tarea inversa a la propuesta en el ejemplo de la Figura 2, o en el apartado c) de la Figura 3, podría partir de fijar el volumen o el área total del cilindro y preguntarse por las medidas del radio de su base y de su altura. La búsqueda de solución a esta tarea: (1) trasciende el empleo netamente aritmético de las fórmulas, ya que requiere analizar la relación funcional entre su volumen o su área, y las medidas del radio de su base y su altura; y (2) admite un conjunto infinito de soluciones para dichas medidas, lo que la convierte en una tarea abierta, pues corresponde a quien la aborda decidir qué criterios utilizar para seleccionar una o más soluciones.

A lo largo del texto, la propuesta de un nuevo tipo de tareas siempre lleva consigo la explicación de la técnica que lo resuelve. Luego se plantean tareas del mismo tipo donde se espera que los alumnos utilicen la misma técnica ya mostrada. Los modelos geométricos, en este caso, los cuerpos geométricos, siempre vienen dados de antemano. La actividad siempre se desarrolla dentro del mundo de las matemáticas y, por tanto, los cuerpos geométricos nunca se presentan como modelos matemáticos. La mayor parte de los tipos de tareas que aparecen en el texto requieren realizar solo cálculos aritméticos. Los tipos de tareas que se proponen no solo tienen solución única, sino que además la técnica empleada para su resolución es única. No se proponen, en ningún caso, tipos de tareas abiertas. Los ostensivos utilizados son siempre del mismo tipo. De modo que se utilizan siempre los mismos símbolos para designar los mismos elementos, $\boldsymbol{V}$ se usa para designar el número de vértices y, posteriormente, para designar el volumen de un cuerpo, $\boldsymbol{A}$ se utiliza para designar el número de aristas y, posteriormente, $\boldsymbol{A}_{\boldsymbol{l}}$ para designar el área lateral y $\boldsymbol{A}_{\boldsymbol{t}}$ para designar el área total. Se emplean $\boldsymbol{r}$ y $\boldsymbol{R}$ para el radio dependiendo de cada caso, $\boldsymbol{h}$ para la altura, etc. Las técnicas utilizadas no aparecen justificadas. En general podemos 
afirmar que la actividad matemática desarrollada es muy poco flexible y las cuestiones a las que responden los saberes presentados son siempre internas al propio modelo geométrico mostrado. No aparecen tareas de modelización espacio-geométrica.

El tipo de actividad matemática en esta unidad cumple las cinco conjeturas con que Fonseca (2004) califica que la actividad matemática escolar en secundaria tiene carácter rígido y atomizado: a) las técnicas matemáticas utilizadas dependen fuertemente de los ostensivos utilizados; b) la aplicación de una técnica no requiere necesariamente de su justificación; c) para cada tarea siempre se privilegia el uso de una única técnica asociada; d) no se presenta la ocasión de utilizar técnicas inversas para resolver tareas matemáticas inversas; y e) no se presentan tipos de tareas abiertas de modelización.

\section{Restricciones institucionales detectadas a partir del análisis del texto escolar}

La TAD define la didáctica como la ciencia de las condiciones y restricciones de la difusión social de las praxeologías. Dentro de la TAD se suele hablar en general de condiciones, pero se consideran también restricciones a aquellas condiciones que son difícilmente modificables desde una posición institucional concreta. De este modo, se dice que una condición es una restricción para una persona, que ocupa un lugar en una institución, cuando dicha persona no tiene la capacidad de cambiarla (con ciertos límites) (Chevallard, 2010). Así, dentro de la institución de la ESO se presentan ciertas restricciones, ligadas a su propio funcionamiento, que los sujetos de dicha institución (profesores y alumnos) no pueden modificar, como el currículo o el tiempo de clase, etc.

En este caso, nos centraremos en el siguiente tipo de restricciones ligadas a lo que Bosch et al. (2004) denominan indicadores del grado de completitud de una organización matemática y que están muy relacionadas con la organización matemática propuesta al realizar la transposición didáctica de la geometría 3D en el texto escolar utilizado por los alumnos:

- La casi total ausencia de tareas inversas en el proceso de estudio que propone el libro de texto. La propuesta de tareas inversas favorece la necesidad de considerar las fórmulas como modelos algebraicos o modelos funcionales, tal como se puede comprobar en el proceso de estudio planteado en el desarrollo del REI.

- La ausencia de tareas abiertas en el estudio de la geometría que se propone en el libro de texto. Este hecho favorece que en el desarrollo del REI los alumnos tiendan a cerrar la tarea y que no consideren el conjunto de soluciones posibles cuando tienen una ecuación con dos incógnitas.

- La falta de cuestionamiento y de justificación tanto de las técnicas empleadas como de los resultados obtenidos. Esta ausencia viene motivada porque las técnicas siempre vienen dadas de antemano y no se da lugar a que las fórmulas sean construidas por los alumnos.

- El planteamiento casi exclusivo de tareas que requieren el uso de las fórmulas como programas aritméticos de cálculo y la ausencia de tipos de tareas que requieran una interpretación algebraico-funcional de las fórmulas.

- La insuficiencia de tareas que provoquen la necesidad de relacionar y articular la geometría 2D y 3D (Rojas y Sierra, 2021).

- La escasez de tareas que requieran utilizar técnicas que hagan necesario relacionar la geometría con el álgebra y las funciones. 
Y, en general, se caracteriza por la ausencia de una problemática de modelización espacio-geométrica en el estudio de la geometría, donde los saberes geométricos surjan como modelos que dan una buena respuesta a los problemas espaciales.

A continuación, presentamos una breve descripción del diseño, experimentación y análisis de un REI en torno al problema espacial de construcción de envases, donde el tipo de tareas propuesto pretende facilitar el uso de técnicas de modelización.

\section{Descripción y análisis del diseño y experimentación del REI}

\subsection{Descripción y análisis a priori del diseño del REI}

Con el objetivo de hacer frente al fenómeno didáctico de la ausencia de técnicas de modelización espacio-geométrica en el estudio de la geometría, nos hemos propuesto llevar a cabo un REI que se ha desarrollado a lo largo de 27 sesiones de 55 minutos (dos sesiones semanales) en una asignatura optativa denominada Ampliación en matemáticas, ofrecida a estudiantes de $3 .^{\circ}$ de ESO, en un instituto de Madrid capital. Asistieron y participaron regularmente 7 alumnos. Siguiendo la metodología de trabajo característica de los REI, propusimos a los estudiantes la formación de dos grupos de trabajo, cuyos componentes fueron elegidos al azar. Los estudiantes $\left(E_{1}, E_{2}, E_{3}\right)$ se asignaron al grupo $A\left(G_{A}\right)$ y los estudiantes $\left(E_{4}, E_{5}, E_{6}, E_{7}\right)$ al grupo $B\left(G_{B}\right)$.

\section{Tipos de tareas propuestas en el REI}

Durante la implementación del REI propusimos cuatro actividades a los estudiantes. Las tres primeras ocuparon la mayor parte del tiempo y dieron origen a una serie de subtareas que emergieron, necesariamente, en el proceso de búsqueda de una respuesta a dichas actividades. La cuarta actividad consistió en un examen individual, donde se evaluaron los conocimientos elaborados en la resolución de las tareas anteriores.

El primer tipo de tarea pretendía el análisis y descripción de las formas de algunos de los envases comerciales que actualmente se usan para envasar, por ejemplo, líquidos como zumo, leche, etc., o sólidos como patatas fritas o chocolates. El segundo tipo consistió en el diseño y construcción de un envase con capacidad para un litro y, el tercero, en el diseño e informe de elaboración de un envase adecuado para un perfume.

En la Figura 4 presentamos un mapa en el que hemos reunido algunas de las cuestiones surgidas durante la implementación de los tres primeros tipos de tareas del REI. En este estudio solo trataremos los tipos de tareas que se corresponden con $Q_{3}$ y $Q_{4}$.

Las tareas que hemos propuesto se caracterizan por su naturaleza progresivamente abierta e inversa. Es decir, por ser tareas en las que, en tanto que abiertas, no se presenta una técnica de solución preestablecida y no hay un listado de datos enunciados a priori que encajen perfectamente con las incógnitas del problema; y en tanto que inversas, los datos y las incógnitas, habitualmente tratados, se invierten. Son tareas en las que la búsqueda de posibles soluciones abre la puerta al uso de modelos algebraicos y/o funcionales. El planteamiento de este tipo de tareas pretende también analizar hasta qué punto es posible superar algunas de las restricciones institucionales para el uso de técnicas de modelización, debidas al tipo de actividad matemática propuesta en el libro de texto. 


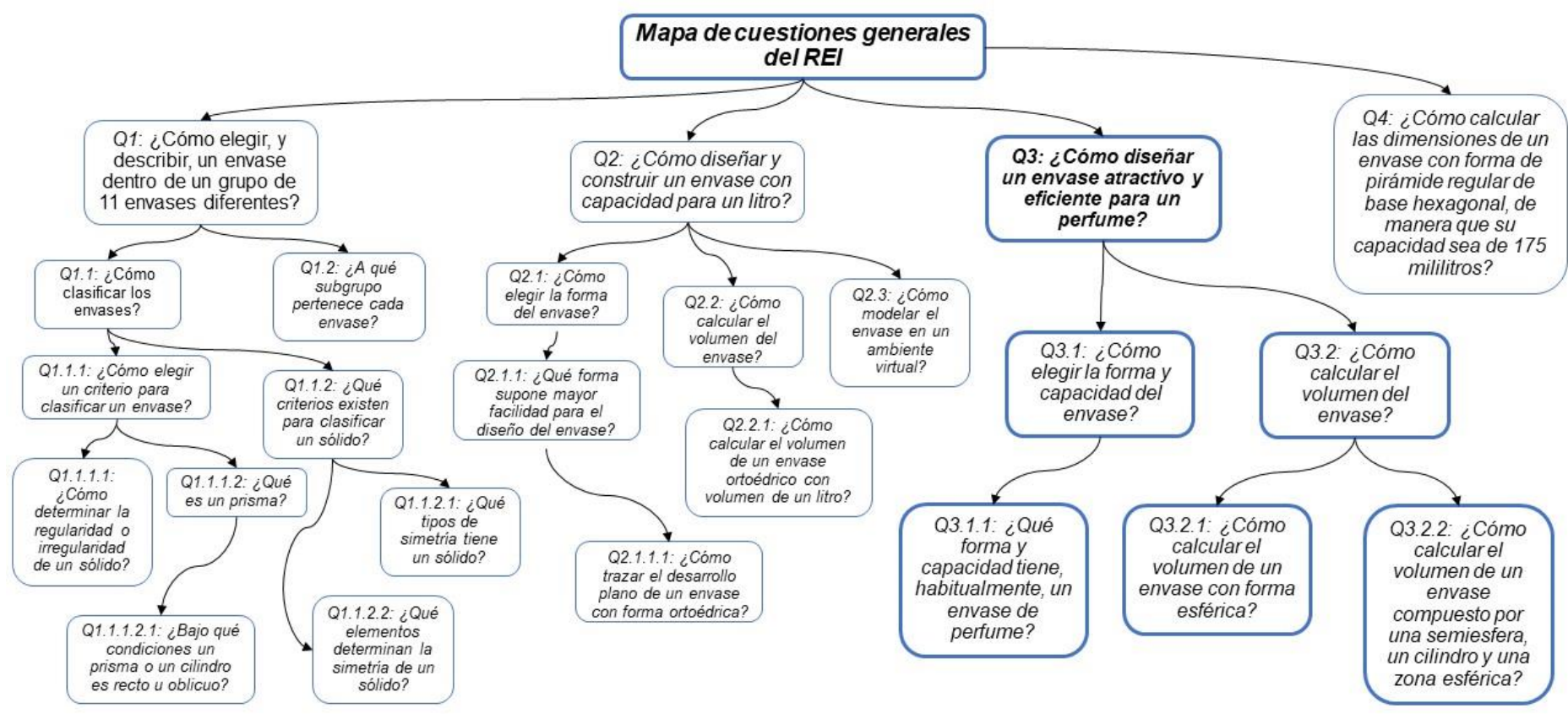

Figura 4. Mapa de cuestiones del REI implementado

\section{Análisis a priori de la tarea de evaluación propuesta}

Para evaluar los conocimientos adquiridos por los alumnos en el REI les propusimos la siguiente tarea:

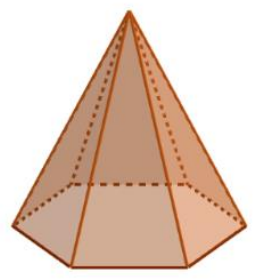

Un equipo consultor ha diseñado un envase con forma de pirámide regular de base hexagonal, con capacidad para 150 mililitros ( $\mathrm{ml}$ ), como se muestra en la figura de la izquierda. Sin embargo, el jefe de producción de la empresa que solicitó el diseño necesitaba que dicho envase, con la misma forma, tuviese una capacidad de 175 $\mathrm{ml}$. Por tanto, se nos ha encomendado la tarea de calcular las medidas que debería tener este envase para que su capacidad sí sea de $175 \mathrm{ml}$, y el área superficial para poder saber la cantidad de papel necesaria que permita trazar su desarrollo plano.

Figura 5. Tarea de evaluación propuesta

En esta actividad el modelo geométrico ya viene dado de antemano, porque nuestro objetivo era comprobar si los alumnos eran capaces de elaborar modelos algebraico-funcionales. La primera tarea propuesta era $t$ : Calcular las dimensiones de una pirámide hexagonal regular con un volumen de $175 \mathrm{ml}$. Esta tarea es de carácter inverso a la planteada en el texto escolar, donde se proporcionan las dimensiones y se pide calcular el volumen. Además, $\boldsymbol{t}$ es una tarea abierta, pues tiene infinitas soluciones y la estrategia para resolverla requiere plantear primero la ecuación con dos incógnitas siguiente: $\mathbf{1 7 5}=\frac{\mathbf{1}}{\mathbf{3}} \boldsymbol{B} \boldsymbol{H}$, donde $\boldsymbol{B}$ es el área de la base y $\boldsymbol{H}$ la altura de la pirámide, y luego la ecuación $\boldsymbol{B}=\frac{\boldsymbol{p} \boldsymbol{a}}{\mathbf{2}}$, siendo $\boldsymbol{p}$ el perímetro de la base y $\boldsymbol{a}$ la apotema de la base.

Una técnica posible para calcular las dimensiones del envase con un volumen de $175 \mathrm{~cm}^{3}$ requiere usar dichas fórmulas como modelos funcionales. Así, una vez obtenido que $\boldsymbol{a}=\frac{\sqrt{3}}{2} \boldsymbol{l}$ mediante el teorema de Pitágoras, y utilizando dichas fórmulas, llegamos a que: $\mathbf{1 7 5}=\frac{\sqrt{3}}{2} \boldsymbol{l}^{2} \boldsymbol{H}$. En dicha ecuación podemos expresar $\boldsymbol{l}$ en función de $\boldsymbol{H}$ o $\boldsymbol{H}$ en función de $\boldsymbol{l}$, así: 


$$
\text { Caso 1: } \sqrt{\frac{\mathbf{3 5 0}}{\boldsymbol{H} \sqrt{3}}}=\boldsymbol{l} \quad \text { Caso } 2: \frac{\mathbf{3 5 0}}{\boldsymbol{l}^{2} \sqrt{\mathbf{3}}}=\boldsymbol{H}
$$

Utilizando GeoGebra, podemos representar gráficamente estas funciones y algunos ejemplos de posibles pirámides con un volumen de $175 \mathrm{~cm}^{3}$ (Figura 6).

Dichas gráficas permiten ver que existe un conjunto infinito de valores de la altura y del lado de la base de la pirámide que dan un volumen de $175 \mathrm{~cm}^{3}$. Ambas gráficas permiten representar de dos maneras diferentes la relación entre $\boldsymbol{l}$ y $\boldsymbol{H}$. Se podrá analizar que dicha relación es inversamente proporcional y que hay algunos valores para los que el diseño del envase se torna poco práctico, como cuando $\boldsymbol{l}$ o $\boldsymbol{H}$ tienden a cero o a infinito, o que no tienen sentido, como los valores negativos en el caso 2.
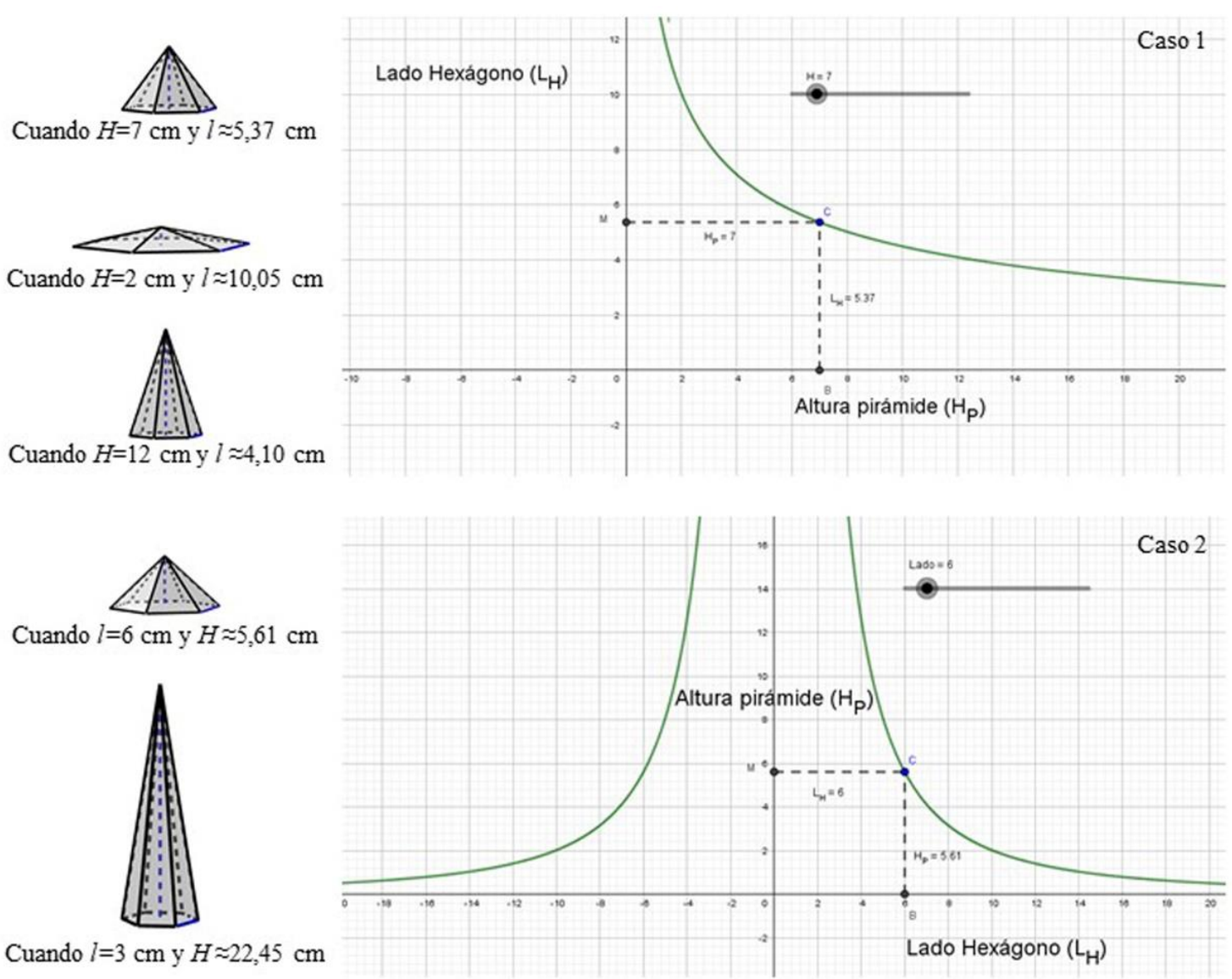

Figura 6. Gráficas del caso 1 y del caso 2 con posibles pirámides solución

El tipo de tarea planteado conduce a que sea necesario considerar las fórmulas del cálculo de volúmenes y de áreas como modelos algebraicos o funcionales, lo que facilita encontrar resultados como el anterior. Una vez consideradas las diferentes soluciones posibles y calculada previamente la apotema de la pirámide, se podrá calcular fácilmente su área total sustituyendo los valores obtenidos en la fórmula correspondiente.

En el siguiente apartado pretendemos describir y analizar la actividad matemática desarrollada por los alumnos durante el REI.

\subsection{Descripción y análisis del proceso seguido para resolver el tercer tipo de tarea}

Una vez que los estudiantes abordaron los dos primeros tipos de tareas del REI, y presentaron a sus compañeros las posibles soluciones a dichas tareas, en la sesión 13 
sugerimos el tercer tipo de tarea a partir de la cuestión: $Q_{3}$ : ¿Cómo diseñar un envase atractivo y eficiente para un perfume? El primer tipo de tareas asociadas a $Q_{3}$ consiste en: $T_{3}$ : Diseñar y realizar un informe para construir un envase adecuado para un perfume con una determinada capacidad o volumen. En este tipo de tareas, a diferencia del segundo, los estudiantes debían definir no solo la forma, sino también la capacidad, lo que la convierte en una tarea más abierta. Al finalizar, los estudiantes tuvieron que exponer a sus compañeros los resultados obtenidos.

El profesor inició su propuesta repartiendo a los alumnos el siguiente texto:

En la compañía de perfumes Afrodita se ha decidido lanzar una nueva fragancia con ocasión de su décimo cuarto aniversario. Para ello, se quiere diseñar un envase atractivo y eficiente para dicho perfume. Nosotros, que ya hemos tenido nuestra primera experiencia como empresa consultora para la compañía envasadora de zumos naturales de España, Zumoluna, ahora vamos a diseñar para la compañía Afrodita el envase que necesitan. Por tanto, como empresa consultora que somos, debemos entregar junto con el diseño del envase, un informe donde: (a) se especifique por qué se ha elegido dicho envase y las características de su diseño, (b) se incluyan los planos del envase si es posible, (c) se explique su proceso de construcción, y (d) se argumente por qué dicho envase es el mejor que podemos sugerir a la compañía de perfumes Afrodita.

Una vez leído el texto, se solicitó a cada grupo de trabajo que elaborase un mapa de cuestiones y posibles respuestas (Figura 7), que les ayudase a marcar un camino en la búsqueda de una posible solución. En dichos mapas, cada grupo estableció algunas subtareas que, a medida que avanzaba el estudio, fueron modificando.
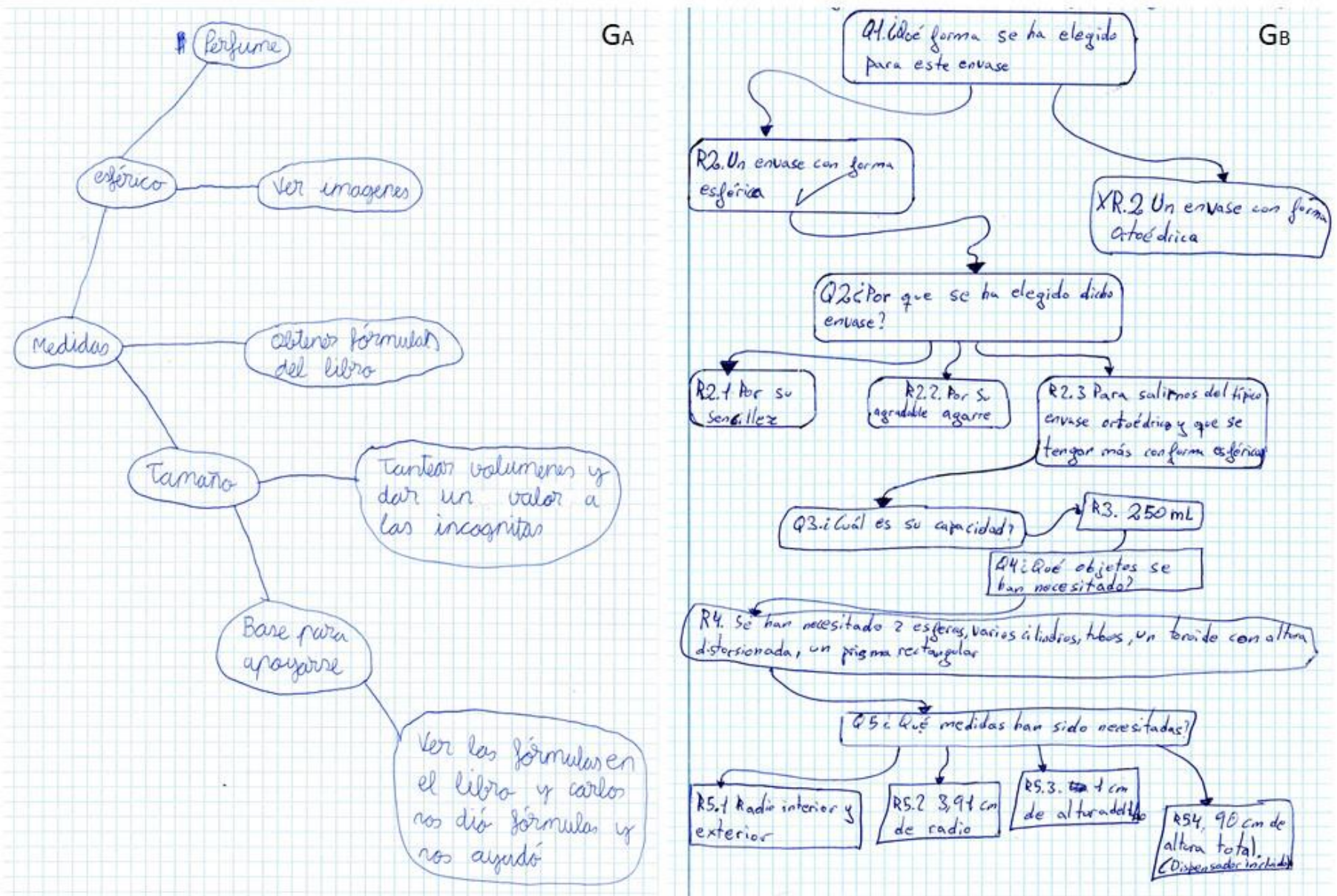

Figura 7. Mapas iniciales de cuestiones y respuestas de estudiantes 
Solo describiremos cómo los estudiantes de $G_{A}$ abordaron las subtareas propuestas. Para resolver $T_{3}$ los alumnos de $G_{A}$ utilizaron la técnica $\tau_{3}$ : Buscar información en internet y en el libro de texto. Para decidir la forma del envase los alumnos eligieron qué modelo de cuerpos geométricos podían utilizar para elaborarlo y para decidir la capacidad utilizaron los prototipos habituales encontrados en internet. En concreto, una forma como la de la Figura 8 y una capacidad de $100 \mathrm{ml}$.

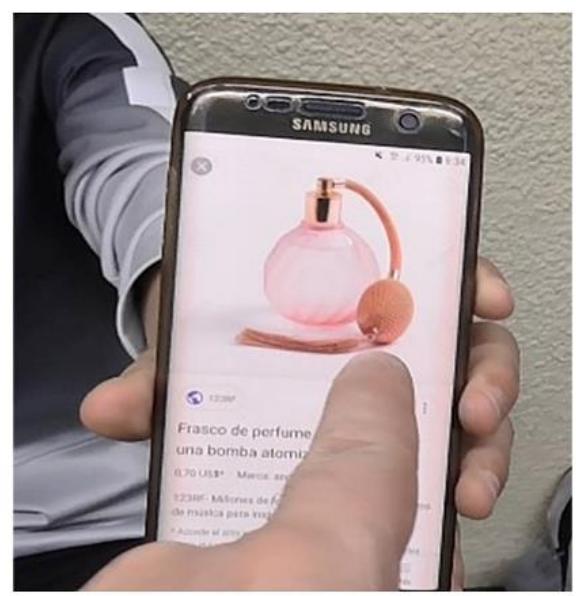

Figura 8. Tipo de envase elegido por los estudiantes

Una vez definida la capacidad del envase, para calcular sus dimensiones decidieron acudir al libro de texto donde aparecía cómo calcular el área de una zona y de un casquete esférico (Figura 9).

Área y volumen de la esfera y de las partes de la esfera

$$
A=4 \pi \cdot R^{2} \quad V=\frac{4}{3} \pi \cdot R^{3}
$$

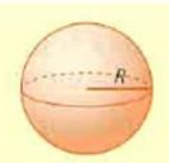

Las áreas de la zona esférica y del casquete esférico de una esfera corresponden al área lateral del trozo de cilindro que la envuelve.

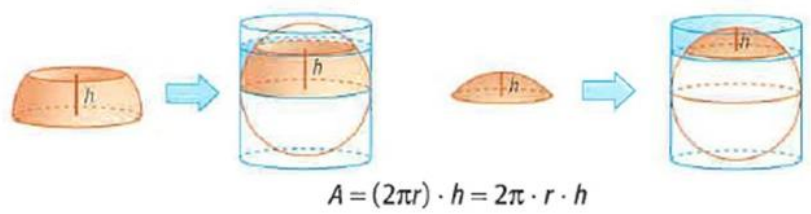

Figura 9. Información sobre el estudio de la esfera en el manual (Alcaide et al., 2016, p. 199)

Dado que los estudiantes ya habían definido la forma y la capacidad del envase, les pedimos que trazaran el boceto del envase y calculasen sus dimensiones. En este punto insistimos porque, inicialmente, pensaban buscar en internet un envase que tuviese la forma y capacidad elegida para así consultar sus medidas. Después, los estudiantes decidieron cambiar la capacidad del envase de $100 \mathrm{ml}$ a $125 \mathrm{ml}$, porque pensaban que era más estético que el envase fuera un poco más alargado que la esfera.

La decisión de cambiar la capacidad del envase vino acompañada de un cambio en la forma. Decidieron que el envase estaría conformado por dos semiesferas, eliminando un casquete esférico a una de ellas, y un cilindro (Figura 10). Esto, les condujo a revisar en su libro de texto cómo calcular el volumen de un cilindro. Cuando preguntamos la razón 
de dicha elección, respondieron que repartían el volumen entre las tres piezas, $50 \mathrm{~cm}^{3} \mathrm{a}$ la primera semiesfera, $25 \mathrm{~cm}^{3}$ al cilindro y $50 \mathrm{~cm}^{3}$ a la otra semiesfera.

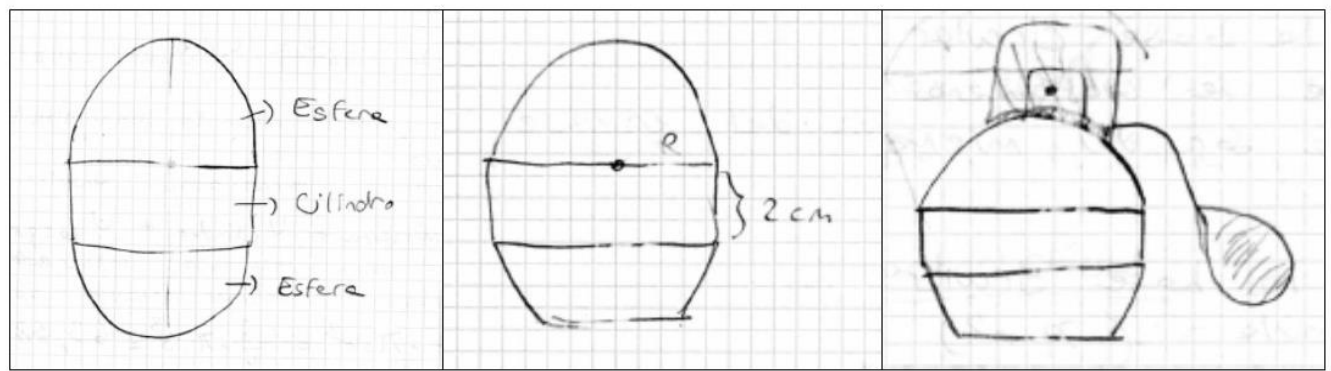

Figura 10. Boceto de izquierda a derecha, diseño inicial, diseño mejorado y diseño final

Tras realizar el diseño del envase, deciden de nuevo cambiar los volúmenes de cada pieza y atribuir $50 \mathrm{~cm}^{3}$ a cada semiesfera y $50 \mathrm{~cm}^{3}$ al cilindro, al pensar que era mejor idea que todas las piezas tuvieran $50 \mathrm{~cm}^{3}$ de volumen. Entonces, deciden resolver la tarea t31: Diseñar un envase para un perfume con una capacidad ${ }^{1}$ o volumen de $150 \mathrm{ml}$ con una forma compuesta por una semiesfera, un cilindro y una zona esférica. Esta t31 es una tarea abierta (se desconocen la técnica que permite resolverla y algunos datos necesarios y suficientes para llevarla a cabo) e inversa respecto de las tareas propuestas en el libro. Para diseñar el envase deciden resolver t311: Calcular las dimensiones de un envase con forma compuesta de semiesfera, cilindro y zona esférica (semiesfera a la que se le ha quitado un casquete esférico) y volumen de $150 \mathrm{~cm}^{3}$, donde el radio de la semiesfera, el radio de la base del cilindro y el radio mayor de la zona esférica son iguales a $\boldsymbol{R}$.

La resolución de $\boldsymbol{t}_{311}$ requiere construir una técnica nueva y compleja porque se deben calcular posibles valores de $\boldsymbol{R}, \boldsymbol{H}, \boldsymbol{r}$ y $\boldsymbol{h}$ (donde $\boldsymbol{R}$ es el radio de la esfera, $\boldsymbol{H}$ es la altura del cilindro, $\boldsymbol{r}$ es el radio del casquete esférico o el radio menor de la zona esférica y $\boldsymbol{h}$ es la altura del casquete esférico) con las ecuaciones:

$$
\begin{gathered}
\frac{2}{3} \pi R^{3}+\pi R^{2} H+\frac{2}{3} \pi R^{3}-\frac{1}{3} \pi h^{2}(3 R-h)=150 \\
R=\frac{r^{2}+h^{2}}{2 h}
\end{gathered}
$$

$\mathrm{Al}$ querer resolver $\boldsymbol{t}_{\mathbf{3 1 1}}$, la primera dificultad de los alumnos fue no considerar que el radio $\boldsymbol{R}$ era igual para los tres componentes del envase. Entonces propusimos:

$\mathrm{P} \quad[\ldots]$ ¿Por qué no pensar en una fórmula para todo el envase? ¿Cómo lo haríamos?

$E_{1} \quad$ Sumando $4 / 3 \pi \boldsymbol{R}^{3}$ y $\boldsymbol{\pi} \boldsymbol{R}^{2} \boldsymbol{h}$. Lo primero es descubrir el radio. Si descubrimos el radio ya podemos empezar a despejar. Y después de tener el radio, calculamos de cuánto queremos la altura $\boldsymbol{h}$ del cilindro ${ }^{2}$.

Las respuestas de $E_{1}$ apuntaban hacia la suma de las fórmulas para calcular el volumen de la esfera y del cilindro. Sin embargo, aunque insistimos en que tratasen de unificar dichas fórmulas, los alumnos optaron por resolver la tarea por trozos simplificando e intentando cerrar la tarea.

\footnotetext{
${ }^{1}$ Aunque en el proceso sí se discutió sobre la diferencia entre volumen y capacidad, los alumnos no lo tuvieron en cuenta en el momento de resolución, identificando el volumen con la capacidad.

${ }^{2}$ Aquí los estudiantes utilizan el símbolo $\boldsymbol{h}$ para designar la altura del cilindro, en lugar de $\boldsymbol{H}$ para luego poderla distinguir de la altura del casquete esférico.
} 
P ¿Cómo haríamos para obtener el radio de la esfera y la altura del cilindro?

$E_{1}[\ldots]$ Despejando equis y encontraríamos el radio [señalando la expresión $\frac{\mathbf{4}}{\mathbf{3}} \pi \mathbf{x}^{3}=\mathbf{1 0 0}$ escrita en su cuaderno]. $Y$, después de encontrar el radio $\boldsymbol{R}$ ya podríamos hacer $\boldsymbol{V}=\boldsymbol{\pi} \boldsymbol{R}^{2} \boldsymbol{h}$ [señalándola en el manual], y despejaríamos $\boldsymbol{h}$. $O$ sea, sería $\boldsymbol{\pi}$ por el radio [conseguido de $\frac{4}{3} \pi \mathbf{x}^{3}=100$ ], al cuadrado, y pondríamos $\mathbf{5 0}=\boldsymbol{\pi} \boldsymbol{R}^{2} \boldsymbol{h}$. Entonces pondríamos la altura como equis, y despejaríamos.

Dicho esto, los alumnos han considerado las dos semiesferas juntas como si fuera una sola esfera y llamando $x$ al radio $R$ de la esfera han obtenido la ecuación $\frac{4}{3} \pi x^{3}=100$. Así, han obtenido $\boldsymbol{R}=\mathbf{2 , 8 8}$, que luego aproximan a $3 \mathrm{~cm}$ (Figura 11), aunque, para el cálculo de la altura del cilindro, usan la medida de $2,88 \mathrm{~cm}$ del radio, obteniendo $1,92 \mathrm{~cm}$ (Figura 12), que aproximan a $2 \mathrm{~cm}$. En los dos casos los alumnos se han visto obligados a considerar la fórmula correspondiente como una ecuación con una única incógnita.

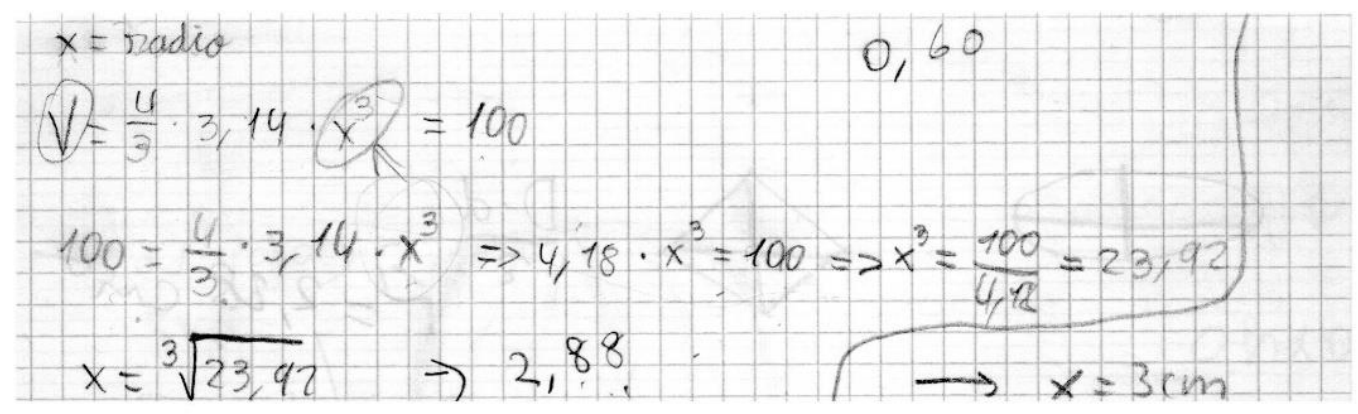

Figura 11. Procedimiento usado por los estudiantes para calcular el radio de la esfera

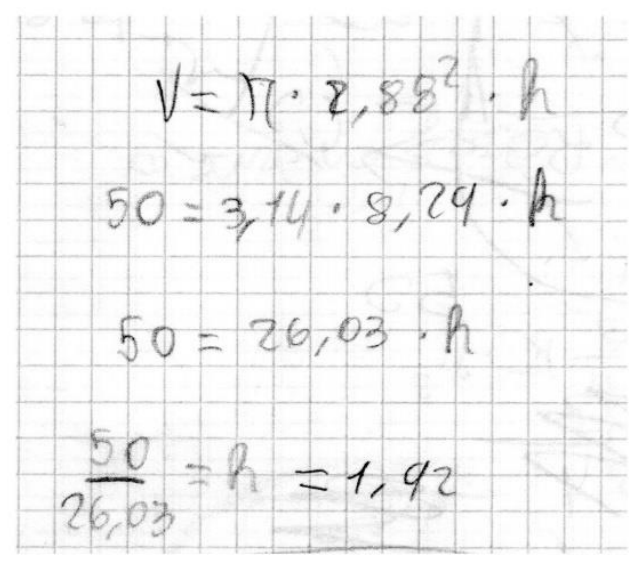

Figura 12. Procedimiento usado por los estudiantes para calcular la altura del cilindro

Luego, los estudiantes han calculado que el volumen de las dos semiesferas era $113,04 \mathrm{~cm}^{3}$ y el del cilindro era $56,52 \mathrm{~cm}^{3}$, con los datos obtenidos, aproximando el radio a $3 \mathrm{~cm}$ y la altura del cilindro a $2 \mathrm{~cm}$. Así, el volumen del envase sin quitar el casquete esférico era 169,56 $\mathrm{cm}^{3}$ (Figura 13). 


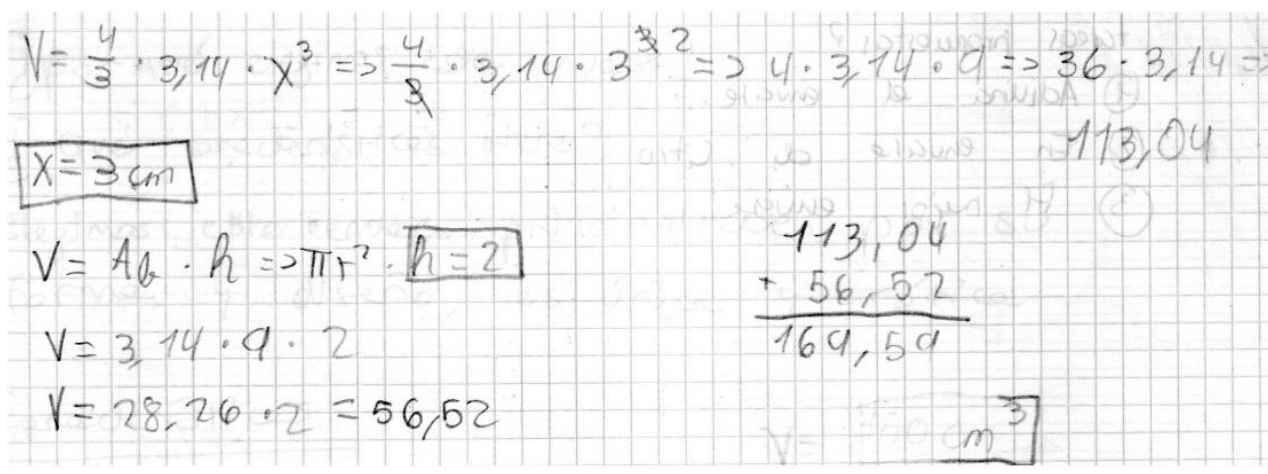

Figura 13. Procedimiento inicial usado por los estudiantes para calcular el volumen del envase

Como el volumen total del envase sobrepasaba los $150 \mathrm{~cm}^{3}$, preguntamos qué volumen debería tener el casquete esférico para que el volumen del envase se ajustase al elegido. Los estudiantes revisaron la información que aparece en el libro sobre cómo calcular el volumen de un casquete esférico, pero, como no la hallaron y no sabían cómo hacerlo, consideramos pertinente cuestionarles sobre la relación que existe entre el radio de la esfera y el radio y la altura del casquete esférico. Fue así como, esbozamos un gráfico (Figura 14) en el que se podía percibir un triángulo rectángulo cuya hipotenusa coincidía con el radio de la esfera $\boldsymbol{R}$, uno de sus catetos medía lo mismo que el radio del casquete esférico $\boldsymbol{r}$, y el otro, el radio de la esfera menos la altura del casquete $\boldsymbol{R}$ - $\boldsymbol{h}$. Entonces surgió la idea de usar el teorema de Pitágoras para calcular $\boldsymbol{r}$ y así determinar si el tamaño de la base era adecuado para el envase (i. e., $\boldsymbol{R}^{2}=\boldsymbol{r}^{2}+(\boldsymbol{R}-\boldsymbol{h})^{2}$ ), y los estudiantes decidieron atribuir una altura ${ }^{3} \boldsymbol{h}$ de $1 \mathrm{~cm}$ para el casquete esférico y así calcularon $\boldsymbol{r}$. Por tanto, en lugar de indagar la relación entre los valores de $\boldsymbol{r}$ y $\boldsymbol{h}$ en la ecuación con dos incógnitas $\mathbf{2} \boldsymbol{R} \boldsymbol{h}-\boldsymbol{h}^{\mathbf{2}}=\boldsymbol{r}^{\mathbf{2}}$ donde $\boldsymbol{R}=\mathbf{3}$, los alumnos decidieron transformarla en una ecuación con una incógnita eligiendo de forma arbitraria $\boldsymbol{h}=\mathbf{1}$ y obtuvieron $\boldsymbol{r}=\mathbf{2 , 2 3}$.
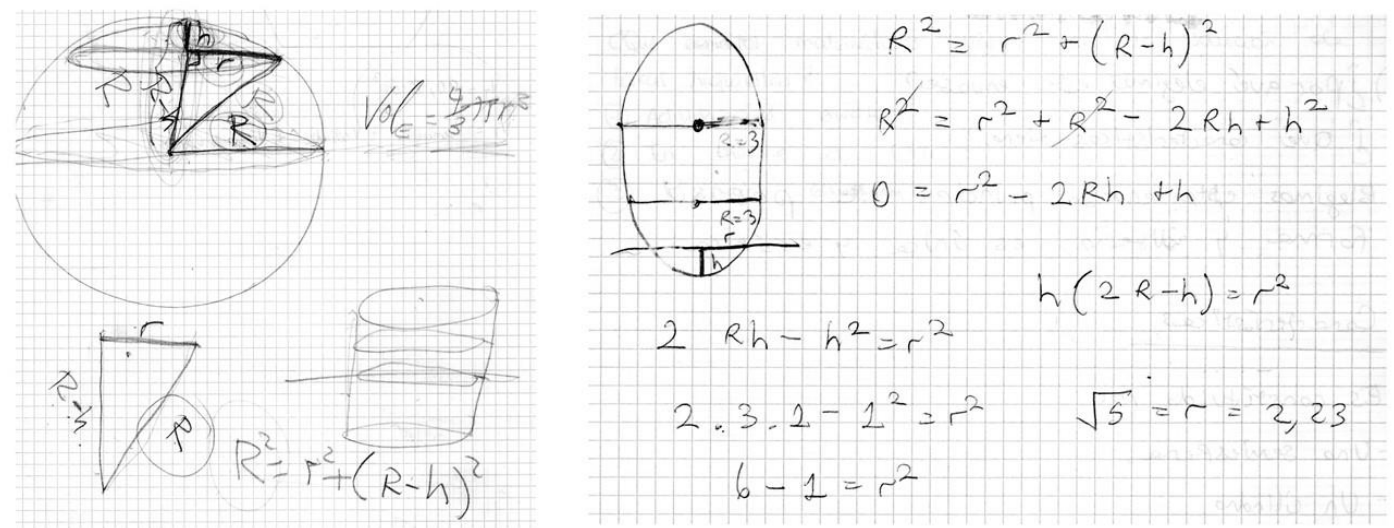

Figura 14. Izquierda, esbozo de la relación entre el radio de la esfera y el radio y la altura del casquete esférico. Derecha, procedimiento usado por los estudiantes para calcular $\boldsymbol{r}$.

Aún hacía falta calcular el volumen del casquete esférico, y como la construcción de una relación que permitiese hacerlo requería de conocimientos más avanzados por parte de los estudiantes, optamos por proporcionarles la fórmula correspondiente:

$$
V=\frac{1}{3} \pi h^{2}(3 R-h)
$$

\footnotetext{
${ }^{3}$ Aquí los alumnos vuelven a utilizar el mismo símbolo $\boldsymbol{h}$ para designar la altura del casquete esférico.
} 
Una vez calculado el volumen del casquete esférico, obtuvieron el volumen del envase sumando los volúmenes de la esfera y del cilindro, y restando el del casquete esférico, es decir, 113,04 +56,52 - 29,3 = 140,26 $\mathrm{cm}^{3}$. Este volumen no coincidía con el previsto de $150 \mathrm{~cm}^{3}$, pero los alumnos no lo cuestionaron. Esto refleja una cierta falta, habitual, de cuestionamiento de los resultados obtenidos por parte de los alumnos.

Al realizar los cálculos se observa una cierta rigidez en el uso de ostensivos, ya que, cuando quieren calcular el radio de la esfera, deciden cambiar la $\boldsymbol{R}$ por la $\boldsymbol{x}$, de modo que las incógnitas en álgebra siempre deben ser designadas por la letra $\boldsymbol{x}$. Igualmente usan siempre la letra $\boldsymbol{h}$ para la altura, ya que es así como siempre se designa en geometría.

El tipo de tarea planteado ha provocado la necesidad de elaborar un modelo geométrico del que fue necesario estudiar sus propiedades. Los alumnos han tenido la necesidad de crear o reformular técnicas, utilizando las fórmulas como ecuaciones algebraicas con varias variables. Ante la dificultad de resolver dichas ecuaciones, han optado por introducir condiciones arbitrarias que simplifican su resolución, tales como, por ejemplo, repartir por igual el volumen total entre los tres componentes del envase y así solo tener que resolver dos ecuaciones con una incógnita cada una, en vez de una ecuación con dos incógnitas, o también, asignar de forma arbitraria un valor a una de las incógnitas cuando se quiere resolver una ecuación con dos incógnitas.

\subsection{Descripción y análisis del proceso seguido para resolver el cuarto tipo de tarea}

Como última actividad del REI propusimos un examen con la tarea de la Figura 5. Solicitamos especialmente que describieran todo el proceso seguido para resolverla. Y proporcionamos las dos fórmulas siguientes que aparecían en su libro: a) volumen de una pirámide $\boldsymbol{V}_{\boldsymbol{p}}=\frac{1}{\mathbf{3}} \boldsymbol{B} \boldsymbol{H}$, siendo $\boldsymbol{B}$ el área de la base y $\boldsymbol{H}$ la altura de la pirámide; y b) área de un polígono regular $\boldsymbol{B}=\frac{\mathbf{1}}{\mathbf{2}} \boldsymbol{p} \boldsymbol{a}$, siendo $\boldsymbol{p}$ el perímetro y $\boldsymbol{a}$ la apotema.

A continuación, presentamos las respuestas del examen que consideramos significativas, dadas por $E_{4}$ y $E_{1}$, pues creemos que nos ayudará a evaluar las dificultades presentadas por los estudiantes para realizar un proceso de modelización.

$E_{4}$ intentó 11 veces calcular las dimensiones de la pirámide y en todos los casos otorgó un valor arbitrario diferente para $\boldsymbol{H}$, a fin de calcular $\boldsymbol{B}$, lo que no le permitió avanzar en el cálculo del área de la pirámide. La Figura 15 presenta su tercer intento.

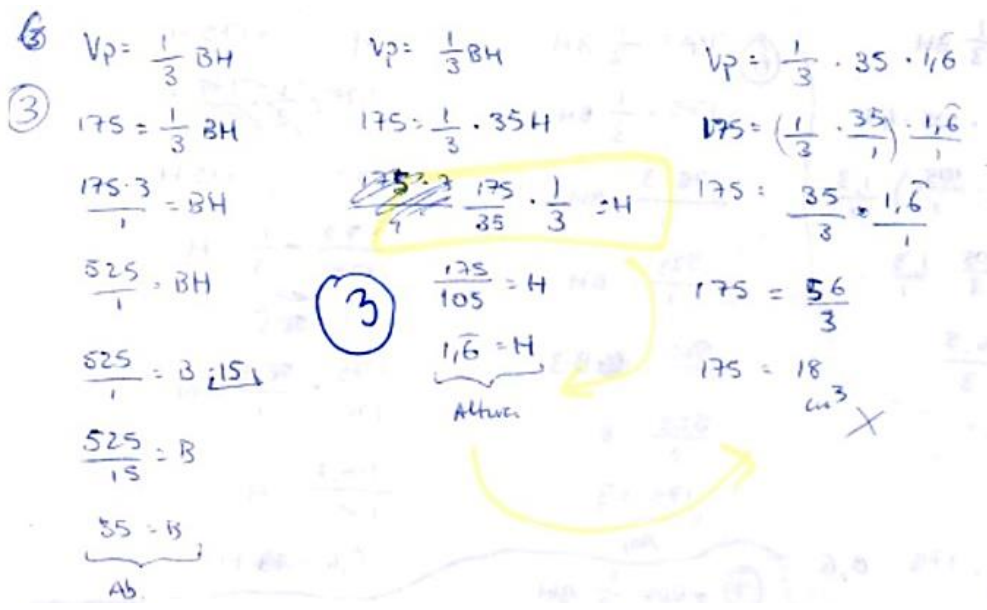

En la $1^{\text {a }}$ columna sustituye $V_{p}$ por $175 \mathrm{y}$ para calcular $B$ decide arbitrariamente dar a $H$ el valor de 15 y obtiene que $B=35$. En la segunda columna decide calcular $H$ y sustituye $V_{p}$ por 175 y $B$ por 35 y obtiene con un mal cálculo que $H$ es $1, \hat{6}$. En la $3^{\text {a }}$ columna sustituye $V_{p}$ por $175, B$ por $35 \mathrm{y}$ $H$ por 1,6 lo que le lleva al absurdo de que $175=18$.

Figura 15. Cálculo de las dimensiones de la pirámide presentado por $E_{4}$ 
En la tarea $\boldsymbol{t}$ planteada, para hallar los posibles valores de $\boldsymbol{H}$ y $\boldsymbol{B}$, debe considerarse que ambos están relacionados. El alumno opta por dar un valor arbitrario a $\boldsymbol{H}$ y así convierte $\mathbf{1 7 5}=\frac{\mathbf{1}}{\mathbf{3}} \boldsymbol{B} \boldsymbol{H}$ en una ecuación con una única incógnita que sí sabe resolver. Luego, para calcular $\boldsymbol{H}$, sustituye el valor de $\boldsymbol{B}$ ya obtenido en la misma ecuación, sin percibir que el valor de $\boldsymbol{H}$ que obtendrá ha de ser el mismo que ya eligió antes.

También queremos mostrar cómo $E_{1}$ calcula el perímetro y la apotema del hexágono regular (Figura 16). Así, $E_{1}$ ha calculado las dimensiones de la pirámide, sin tener en cuenta que los valores de la apotema y del lado del polígono regular son interdependientes. Después con dicha información $E_{1}$ calcula el área total de la pirámide.

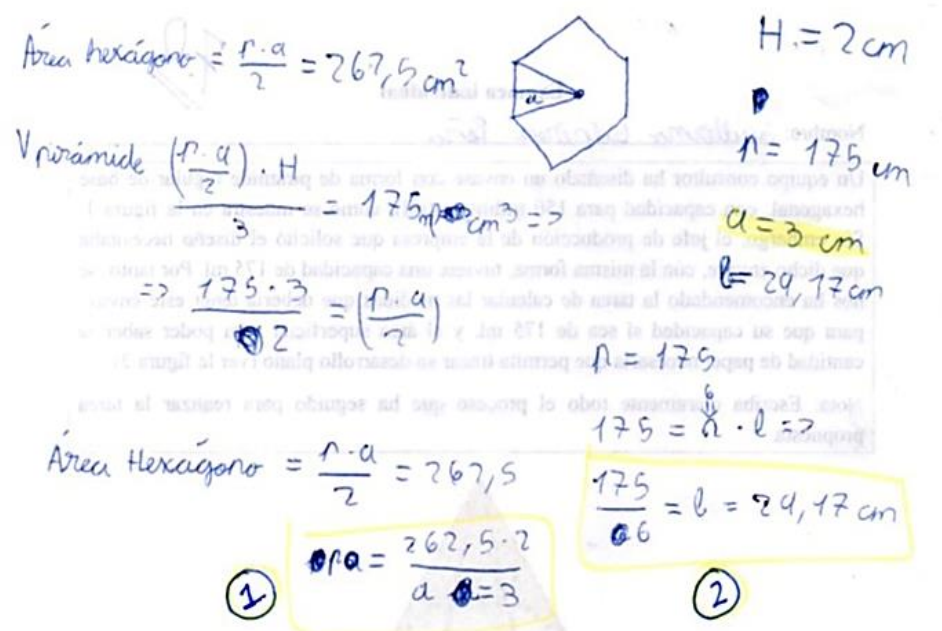

Primero sustituye en la fórmula $B=\frac{1}{2} p a, \mathrm{~B}$ por 262,5 . En la $2^{a}$ fila utiliza la fórmula $V_{p}=$ $\frac{1}{3} B H$ sustituyendo $B$ por $\frac{1}{2} p a$ y elige que $H$ vale 2 de forma arbitraria. Así calcula que $\frac{1}{2} p a=262,5$. Luego elige arbitrariamente que $a=3$ y obtiene que el perímetro $p=175$. $\mathrm{Y}$ termina obteniendo que el lado del hexágono es $l=29,17$, después de dividir 175 entre 6 .

Figura 16. Cálculo de las dimensiones de la pirámide por $E_{1}$

A diferencia del caso anterior, los valores de $\boldsymbol{p}$ y de $\boldsymbol{a}$ están ligados entre sí independientemente de la ecuación planteada, pues siempre $a=\frac{p}{\mathbf{1 2}} \sqrt{\mathbf{3}}$. Aquí, se puede intentar construir la figura y comprobar que no es posible, ya que si $\boldsymbol{p}=\mathbf{1 7 5}$ entonces $\boldsymbol{a} \cong \mathbf{2 5}, \mathbf{2 6}$. Este tipo de error puede venir provocado porque las fórmulas no son construidas por los alumnos, sino que vienen dadas de antemano.

Cabe señalar que la fórmula del área de un polígono regular siempre se presenta en los textos escolares en función del perímetro $\boldsymbol{p}$ y de la apotema $\boldsymbol{a}$, lo que sugiere que ambos datos son independientes. En el caso del hexágono regular la relación es evidente y en los demás polígonos regulares se puede demostrar mediante razones trigonométricas. En definitiva, la presentación escolar del problema del área de un polígono regular da a entender que esta depende de dos variables. Al plantear el problema inverso, determinar el lado dada el área, se pone de manifiesto que el área depende de una sola variable.

Para terminar el análisis del REI experimentado, podemos afirmar que el tipo de tareas propuesto ha permitido que los alumnos hayan llegado a considerar parcialmente las fórmulas como modelos algebraicos. Sin embargo, no ha sido posible que las interpreten y las utilicen como modelos funcionales, entre otros motivos, porque no han podido usar GeoGebra en el aula de clase.

\section{Las restricciones institucionales después de la experimentación realizada}

Después de implementar el REI siguen presentándose ciertas restricciones, ligadas al funcionamiento de la institución de educación secundaria, que profesores y alumnos no pueden modificar, como el tiempo de clase (55 minutos) o la prohibición de usar el móvil 
o el ordenador en clase. Dichas restricciones han limitado la posibilidad de proponer tipos de tareas que serían útiles para conseguir dar sentido a los saberes que se pretende enseñar. Plantear tareas abiertas requiere un tiempo de clase de al menos 90 minutos, que permita que los alumnos trabajen en grupo, discutan cómo abordar la tarea, la lleven a cabo y presenten a la clase su propuesta de resolución. También es necesario usar en el aula recursos disponibles en internet como GeoGebra y Tinkercad. El uso del software dinámico ayudará a dar sentido al uso de los modelos algebraico-funcionales para resolver problemas espaciales. El trabajo con el programa de diseño e impresión en 3D permitirá validar la solución del problema espacial del diseño y elaboración de un sólido.

En lo que se refiere a las restricciones ligadas al grado de completitud de la organización matemática desarrollada en el REI, el tipo de tareas propuesto ha favorecido que los alumnos hayan realizado esbozos de modelos geométricos y algebraicos con el fin de dar respuesta al problema espacial de diseñar un envase para un perfume. Las tareas propuestas en el REI se caracterizan por ser abiertas e inversas y, como se ha mostrado en la experimentación, han provocado la necesidad de considerar las fórmulas como modelos algebraicos que ha permitido relacionar la geometría con el álgebra.

Ha sido posible llevar a cabo la utilización de modelos geométricos y algebraicos debido a que la organización matemática propuesta tiene un mayor grado de completitud que la que ofrece el libro de texto, como se pone de manifiesto en el tipo de tareas propuestas en el REI y en la necesaria elaboración de técnicas de resolución por parte de los estudiantes. Sigue existiendo cierta rigidez en el uso de ostensivos en las actividades desarrolladas en el REI, pero hemos percibido una mayor flexibilidad en la respuesta de los estudiantes a la tarea de evaluación propuesta.

En el desarrollo del REI ha seguido habiendo una cierta dificultad para que los alumnos sean capaces de construir técnicas nuevas y el profesor ha debido insistir para evitar su tendencia a simplificar y cerrar las tareas propuestas, animándolos a elaborar sus propias respuestas. Esta dificultad está ligada a la costumbre que impone el modelo pedagógico dominante, donde las tareas que los alumnos están habituados a resolver requieren un tiempo reducido, pues suelen ser tareas aisladas y cerradas.

El REI implementado se ha realizado de una forma bastante abierta y libre, dejando que los estudiantes tomasen muchas decisiones sin apenas influencia y dirección por parte del profesor. Sin embargo, pensamos que, si nuestro objetivo es conseguir que los estudiantes utilicen técnicas de modelización para la resolución de problemas espaciales y sean capaces de cuestionarlas y justificarlas, es necesario que el profesor intervenga de manera más cercana en el desarrollo del proceso de estudio, favoreciendo que se traten aquellas cuestiones que permiten potenciar, provocar y posibilitar dicho objetivo. Postulamos que un instrumento de gran ayuda para orientar dicha estrategia didáctica será disponer de un modelo epistemológico de referencia en torno a la determinación y construcción de sólidos, que estamos elaborando en este momento.

\section{Conclusiones}

El análisis de la experimentación realizada y del texto escolar utilizado por los alumnos nos permite concluir que es necesario y posible crear las condiciones para que la problemática de modelización espacio-geométrica pueda vivir en la ESO. Para ello, proponemos incidir sobre los modelos didáctico y epistemológico dominantes y vigentes en la enseñanza de la geometría en la ESO, que viene representado por la propuesta que se hace visible tanto en el currículo oficial como en los libros de texto. 
En lo relativo al modelo didáctico creemos necesario cambiar el contrato didáctico habitual, de modo que el profesor pase a ser el director del proceso de estudio y el alumno asuma tareas relativamente ausentes como, por ejemplo, plantear cuestiones, elaborar informes, realizar presentaciones y evaluar el trabajo realizado, entre otras. El modelo que proponemos se materializa en el dispositivo de los REI, donde el saber geométrico debe surgir como respuesta a una cuestión generatriz de gran interés para la comunidad de estudio. Para facilitar la puesta en práctica de este dispositivo será conveniente poder ampliar el tiempo de clase de 55 a 90 minutos con el objetivo de que los alumnos puedan llevar a cabo presentaciones del trabajo realizado en grupo.

En relación con el modelo epistemológico, nuestra propuesta consiste en elaborar un modelo epistemológico de referencia (MER) de la geometría en secundaria, alternativo al vigente. Se trata de un modelo capaz de caracterizar y dar sentido a la actividad geométrica interpretada como una actividad de determinación y construcción de todo tipo de figuras (en particular, de sólidos). Una parte de dicho MER, que hemos empezado a desarrollar, contendrá de manera relevante las cuestiones (y las tareas asociadas) relativas a la problemática de la modelización espacio-geométrica que consideramos como la problemática de iniciación a la geometría en la enseñanza secundaria. Partiendo de tipos de tareas abiertas e inversas, planteados primero en el espacio sensible, como los propuestos en el REI experimentado, el MER proporcionará diversos recorridos posibles, donde utilizando modelos matemáticos de todo tipo y con la ayuda de herramientas (incluyendo GeoGebra), se puedan resolver dichas tareas. El profesor, como director del proceso de estudio, dispondrá de una herramienta valiosa a utilizar en distintos momentos y de forma más o menos directiva, dependiendo de las decisiones de los alumnos a lo largo del REI. Pretendemos superar así algunas restricciones institucionales que siguen presentándose en relación con la problemática espacio-geométrica y, al mismo tiempo, facilitar que los alumnos asuman nuevas responsabilidades.

\section{Agradecimientos}

Proyecto RTI2018-101153-A-C22, Programa Estatal de I+D+i Orientada a los Retos de la Sociedad.

\section{Referencias}

Alcaide, F., Hernández, J., Serrano, E., Moreno, M. y Pérez, A. (2016). Matemáticas orientadas a las enseñanzas académicas. $3^{\circ}$ ESO. SM Savia.

Barquero, B. (2009). Ecología de la modelización matemática en la enseñanza universitaria de las matemáticas. Trabajo de Tesis doctoral sin publicar, Universitat Autónoma de Barcelona. https://www.tdx.cat/handle/10803/3110\#page=1

Barquero, B. (2020). Introducción a "Investigaciones sobre enseñanza y aprendizaje de la modelización matemática: Aproximaciones a la problemática de su diseño, implementación y análisis". AIEM, 17, 1-4. https://doi.org/10.35763/aiem.v0i17.326

Barquero, B., Bosch, M. y Gascón, J. (2013). Las tres dimensiones del problema didáctico de la modelización matemática. Educaçao Matemática Pesquisa, 15(1), 128.

Berthelot, R. y Salin, M. H. (2001). L'enseignement de la géométrie au début du collège. Comment concevoir le passage de la géométrie du constat à la géométrie déductive? Petit $x, 56,5-34$. 
Berthelot, R. y Salin, M. H. (2005). Vers une problématique de modélisation dans l'enseignement elementaire de la géométrie. En M. H. Salin, P. Clanché y B. Sarrazy (eds.), Sur la théorie des situations didactiques (pp. 125-142). La Pensée Sauvage.

Bolea, P. (2002). El proceso de algebrización de organizaciones matemáticas escolares (Trabajo de Tesis doctoral). Editorial Prensas de la Universidad de Zaragoza

Bosch, M., Fonseca, C. y Gascón, J. (2004). Incompletitud de las organizaciones matemáticas locales en las instituciones escolares. Recherches en Didactique des Mathématiques, 24, 1-47.

Chevallard, Y. (2010). Le sujet apprenant entre espace et dispositif. Commentaires depuis la théorie anthropologique du didactique. Communication présentée aux journées du Lisec Gérardmer. http://yves.chevallard.free.fr/spip/spip/IMG/pdf/

Florensa, I., García, F. J. y Sala, G. (2020). Condiciones para la enseñanza de la modelización matemática. Estudios de caso en distintos niveles educativos. AIEM, 17, 21-37. https://doi.org/10.35763/aiem.v0i17.315

Fonseca, C. (2004). Discontinuidades matemáticas y didácticas entre la enseñanza secundaria y la enseñanza universitaria. Trabajo de Tesis Doctoral sin publicar. Universidad de Vigo.

García, F. J. (2005). La modelización como herramienta de articulación de la matemática escolar. De la proporcionalidad a las relaciones funcionales. Trabajo de Tesis Doctoral sin publicar. Universidad de Jaén.

Gascón, J. (1994). El papel de la resolución de problemas en la enseñanza de las matemáticas. Educación Matemática, 6(3), 37-51

Lucas, C. (2015). Una posible «razón de ser» del cálculo diferencial elemental en el ámbito de la modelización funcional. Trabajo de Tesis Doctoral sin publicar. Universidade de Vigo. http://hdl.handle.net/11093/542

Perrin-Glorian, M.-J., Mathé, A. C. y Leclercq, R. (2013). Comment peut-on penser la continuité de l'enseignement de la géométrie de 6 a 15 ans? Le jeu sur les supports et les instruments. Repères IREM, 90, 5-41.

Rojas, C. y Sierra, T. (2017). Análisis del currículo y de manuales escolares para el caso de los conocimientos espaciales y geométricos en la educación secundaria obligatoria. Comunicación presentada en el grupo DMDC, XXI Simposio SEIEM.

Rojas, C. y Sierra, T. (2021). Conocimientos geométricos como respuesta a un problema espacial en el desarrollo de un recorrido de estudio e investigación. Educación Matemática, 33(1), 208-239. https://doi.org/10.24844/EM3301.08

Ruiz-Munzón, N. (2010). La introducción del álgebra elemental y su desarrollo hacia la modelización funcional. Trabajo de Tesis doctoral sin publicar. Universitat Autònoma de Barcelona.

Salin, M. H. (2004). La enseñanza del espacio y la geometría en la enseñanza elemental. En C. Chamorro (ed.), Números, formas y volúmenes en el entorno del niño (pp. 3780). Ministerio de Educación y Ciencia.

Schukajlow, S., Kaiser, G. y Stillman, G. (2018). Empirical research on teaching and learning of mathematical modelling: a survey on the current state-of-the-art. ZDM Mathematics Education 50, 5-18. https://doi.org/10.1007/s11858-018-0933-5 
Serrano, L. (2013). La modelización matemática en los estudios universitarios de economía y empresa: análisis ecológico y propuesta didáctica. Trabajo de Tesis Doctoral sin publicar. Universitat Ramon Llull.

https://www.tdx.cat/handle/10803/101204\#page=1

\section{Referencias de los autores}

Carlos Rojas Suárez, Universidad de Antioquia (Colombia). carlroja@ucm.es

Tomás Ángel Sierra Delgado, Universidad Complutense de Madrid (España). tomass@ucm.es 


\title{
Institutional constraints that hinder spatial-geometric model- ling in secondary school teaching
}

\author{
Carlos Rojas Suárez, Universidad Complutense de Madrid \\ Tomás Ángel Sierra Delgado, Universidad Complutense de Madrid
}

In this paper, we aim to explain institutional restrictions that hinder student mathematical modelling techniques in Compulsory Secondary Education focusing on spatial-geometric modelling. Within the frame of the Anthropological Theory of the Didactic, we postulate that the dominant epistemological-didactic model in the teaching of mathematics, fundamentally represented by the current curriculum, textbooks and classroom practices, gives rise to didactic phenomena linked to the loss of the raison d'être of compulsory secondary school geometry and hinders the possibility of carrying out modelling processes in this institution. We base our explanation on the comparison between: (1) the mathematical activity proposed in the textbook used by 3rd-year students of Secondary Compulsory Education about the determination and construction of solid figures, and (2) the observation and analysis of difficulties emerged when a small group of 3rd-year students faced tasks about the determination and construction of solid figures within a study and research path. In this study process, we introduced the spatial-geometric modelling problem by using a generating question around a spatial task: the design and construction of perfume bottles. From our analysis, we postulate that: (a) the type of tasks proposed provokes the building of geometric models, the study of its properties and the creation (or reformulation) of techniques that should be justified; (b) solving the open and inverse tasks that emerge along the study process leads to considering volume and area formulae as algebraic or functional models rather than arithmetic algorithms. We suggest the necessity of questioning the current dominant epistemological-didactic model in the teaching of geometry in the Compulsory Secondary Education. Regarding the epistemological model, we propose introducing the spatial-geometric modelling problem starting from spatial tasks that will lead to the determination and construction of solid figures. Regarding the didactic model, it will be necessary to change the usual didactic contract so that the teacher becomes the director of the study process, and the students take on new didactic responsibilities such as posing questions, preparing reports and making presentations that allow the assessment of their work. 